\title{
Echogenic Intracardiac Focus: A Sonographic Sign for Fetal Down Syndrome
}

\author{
BRYANN BROMLEY, MD, ELLICE LIEBERMAN, MD, DrPH, LANE LABODA, RT, RDMS, \\ AND BERYL R. BENACERRAF, MD
}

Objective: To determine whether an echogenic intracardiac focus identified in the second-trimester fetus is related to an increased risk of Down syndrome.

Methods: During a 10-month period, all women with singleton gestations who underwent second-trimester genetic amniocentesis for non-imaging indications were evaluated prospectively by prenatal sonography. The presence or absence of an echogenic intracardiac focus was noted. Karyotypic information was obtained on each fetus.

Results: Among the 1334 patients in the study group, 66 fetuses $(4.9 \%)$ had an echogenic intracardiac focus. Four of 22 fetuses $(18 \%)$ with trisomy 21 had an echogenic intracardiac focus, compared with $62(4.7 \%)$ of 1312 fetuses without Down syndrome who also had an echogenic intracardiac focus $(P=.004)$. Sonographic identification of an echogenic intracardiac focus was associated with a fourfold increased risk of Down syndrome (risk ratio $4.3,95 \%$ confidence interval 1.5-12.3). The overall prevalence of Down syndrome in our study population was $1.6 \%$. The sensitivity, specificity, and positive predictive value for using the presence of an echogenic intracardiac focus to identify a fetus with Down syndrome was $18.2,95.3$, and $6.1 \%$, respectively. Extrapolating to a lower risk population, the positive predictive value of an echogenic intracardiac focus for detecting Down syndrome in patients at an age-based risk of one in 250 , one in 500 , and one in 1000 was calculated to be 1.53 , 0.77 , and $0.39 \%$ respectively.

Conclusion: Fetuses with an echogenic intracardiac focus have a significantly increased risk of Down syndrome. Although most fetuses with this finding are normal, patients carrying fetuses with an echogenic intracardiac focus should be counseled about the increased risk of trisomy 21. (Obstet Gynecol 1995;86:998-1001)

An echogenic intracardiac focus within the fetal cardiac ventricle has been identified as a normal variant for many years. ${ }^{1-+}$ Recently, several reports ${ }^{--}$have sug-

From the Departments of Obstetries and Cuntecology and Radioldegly. Massachusetts Gencral Hospital and Brighin is Women's Hospital. Haraard Medical School. Boston. Masinchusetts gested an association between autosomal trisomies and an echogenic intracardiac focus, specifically trisomies 21 and 13. Roberts and Genest ${ }^{8}$ demonstrated that microcalcifications in the human fetal papillary muscle were features seen in fetuses with autosomal trisomies. We sought to determine if an echogenic intracardiac focus in the second-trimester fetal heart was in any way related to an increased risk of Down syndrome.

\section{Materials and Methods}

We identified all women who underwent genetic amniocenteses at 13-21 weeks' gestation from February 1, 1994, through November 30, 1994. 'Those who had an amniocentesis solely because of a structural abnormality seen on an ultrasound scan were excluded from the study, leaving only those who had karyotyping because of standard non-imaging indications, such as advanced maternal age, abnormal serum screening, or a family history of genetic abnormalities. Women carrying multiple gestations were excluded from the final study group.

Before amniocentesis, each fetus was evaluated sonographically, including standard biometry and a structural fetal survey. When technically feasible, the four chambers of the heart and great vessels were evaluated. In each case, the presence or absence of an echogenic focus within the fetal cardiac ventricles was determined. An echogenic intracardiac focus was deemed to be present when the echogenicity in the region of the papillary muscle in either ventricle was comparable to bone (Figures 1 and 2). The location of the echogenic intracardiac focus was recorded as being either in the left or right ventricle. The presence or absence of associated structural fetal abnormalities was determined at the time of the scan. All ultrasound examinations were performed using an Acuson 128 XP-10 (Mountain View, CA) with a variable-focus 3.5- or 5-MHz transducer. 


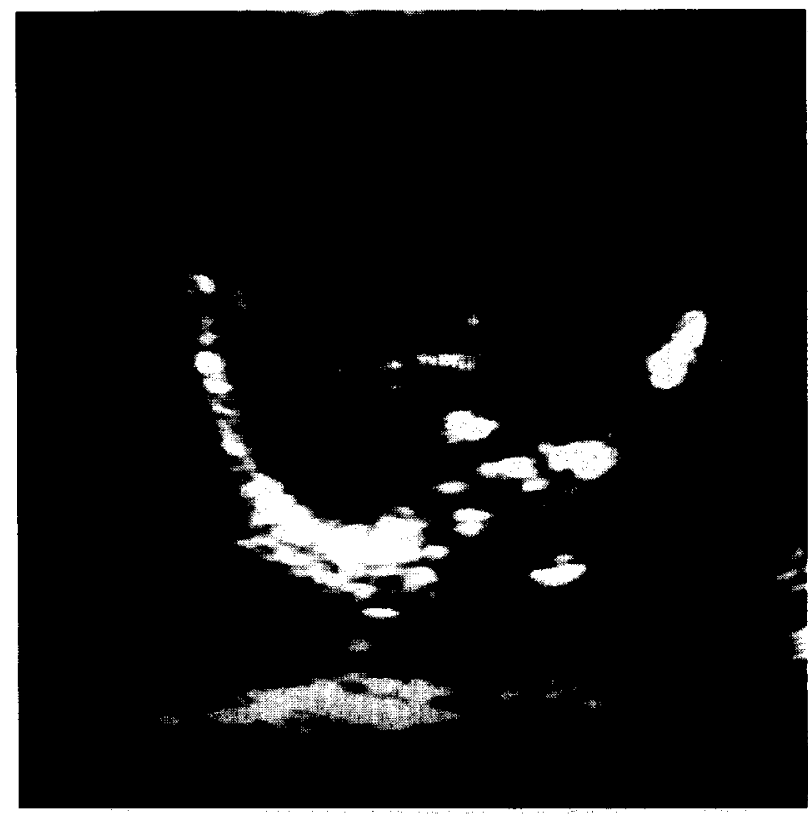

Figure 1. Four-chamber view of the fetal heart showing an echogenic intracardiac focus (arrow) within the left ventricle.

All patients underwent amniocenteses for karyotype, and follow-up was obtained from the cytogenetic laboratories for each fetus in the study. Additional follow-up was obtained from the newborn medical records on each fetus with an echogenic intracardiac focus.

The risk ratio was calculated as the rate of echogenic intracardiac focus among fetuses with Down syndrome divided by the rate of echogenic intracardiac focus among fetuses without Down syndrome. The 95\% confidence intervals $(\mathrm{CI})$ around the risk ratio were also calculated. The significance of the association between echogenic intracardiac focus and Down syndrome was evaluated using $\chi^{2}$ analysis. The sensitivity of echogenic intracardiac focus as a screening test for Down syndrome was calculated as the percentage of infants with Down syndrome who had an echogenic intracardiac focus present. The specificity was defined as the percentage of fetuses without Down syndrome who did not have an echogenic intracardiac focus. The predictive value of a positive test was the percentage of infants with a positive test who had Down syndrome.

\section{Results}

There were 1454 fetuses at 13.21 wecks' gestation who had genetic amniocenteses at our laboratory during the study period. Eighty were excluded from the study because they were referred for amniocentesis only after an abnormal sonogram, leaving 1374 fetuses who were karyotyped for standard non-imaging indications over the course of the 10 -month study. One thousand one hundred fifty-three patients were referred because of advanced maternal age, 145 for abnormal alphafetoprotein, and 76 for otherwise abnormal triple screening or family history of a genetic abnormality. Forty women with twin gestations were excluded from the study group, leaving 1334 singleton fetuses for analysis. Sixty-six of 1334 (4.9\%) fetuses had an echogenic intracardiac focus seen in the fetal heart, $60(91 \%)$ in the left ventricle, five $(7.5 \%)$ in the right ventricle, and one $(1.5 \%)$ in both ventricles.

Four of 66 fetuses $(6 \%)$ with an echogenic intracardiac focus had Down syndrome on karyotype; three of the foci were in the left ventricle and one was in both ventricles. Twenty-two fetuses with Down syndrome were identified karyotypically during the study period. Four $(18 \%$, 95\% CI 2.1-34.3) of these 22 had an echogenic intracardiac focus and 18 did not. This is compared with $62(4.7 \%, 95 \%$ CI 4.1-5.3) of 1312 fetuses without Down syndrome who had an echogenic intracardiac focus ( $P=.004$ ). In our high-risk population, the sonographic identification of an echogenic intracardiac focus was associated with a risk ratio of 4.3 for the occurrence of Down syndrome (95\% CI 1.5-12.3).

The overall incidence of Down syndrome in our study population was $1.6 \%$. Based on this high-risk study population, the sensitivity, specificity, and positive predictive value for using the presence of an echogenic intracardiac focus to identify a fetus with Down syndrome was $18.2,95.3$, and $6.1 \%$, respectively.

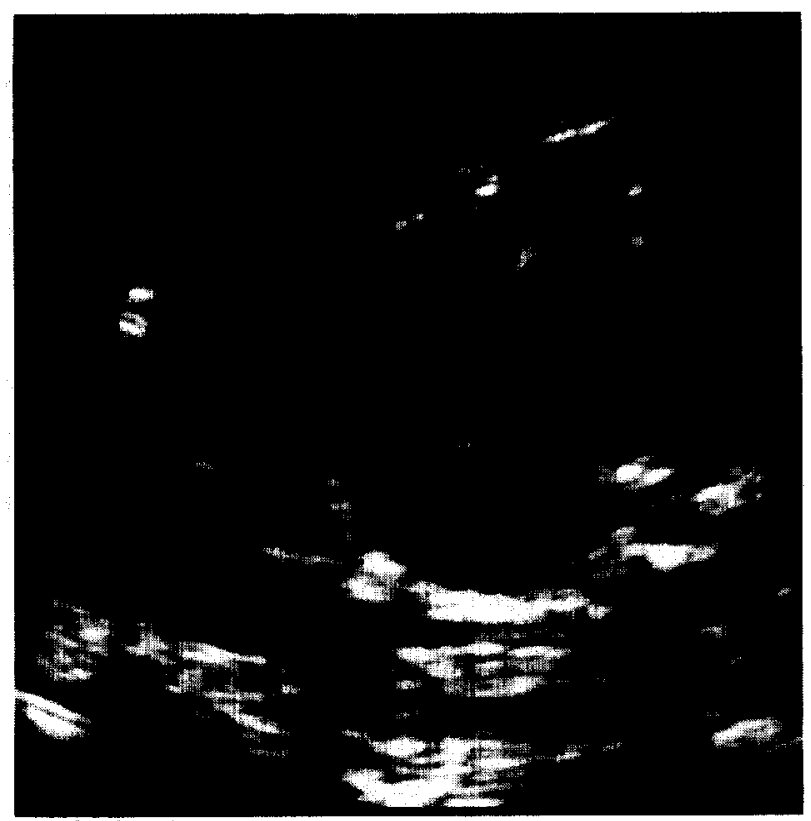

Figure 2. Long-axis view of the heart showing the location of the echogenic intracardiac focus (arrow) within the left ventricle. 
The predictive value of an echogenic intracardiac focus for Down syndrome varies with the prevalence of the disease. Extrapolating to a lower risk population, the positive predictive value of an echogenic intracardiac focus for detecting Down syndrome in patients at an age-based risk of one in 250 , one in 500 , and one in 1000 is $1.53,0.77$, and $0.39 \%$, respectively.

One of the four fetuses with Down syndrome and an echogenic intracardiac focus also had a thickened nuchal fold. An additional fetus with Down syndrome was scanned at 14 weeks, but a complete structural evaluation of the fetus was not possible. The remaining two fetuses with Down syndrome had an echogenic intracardiac focus and no other structural abnormalities on complete sonographic fetal survey. Three of the four fetuses with Down syndrome and an echogenic intracardiac focus were referred for advanced maternal age, and one for a low maternal serum alpha-fetoprotein.

Among the 62 fetuses with echogenic intracardiac focus and normal karyotypes, there were only two fetuses with structural abnormalities, one of whom had gastroschisis and the other a duplicated ureter; both of these were confirmed at birth. One other fetus had hypospadias diagnosed at birth; the condition had not been identified at second-trimester amniocentesis. The remaining 59 fetuses were discharged from the hospital after normal newborn examinations.

\section{Discussion}

An echogenic intracardiac focus has been reported in $0.46-20 \%$ of normal fetuses. ${ }^{1.4}$ However, two studies ${ }^{2.3}$ place the incidence of echogenic intracardiac focus at $3.5 \%$. We found an incidence of $4.9 \%$, which agrees with most reports. Our data confirms the work of Petrikovsky et $\mathrm{al}^{3}$ and How et al, ${ }^{1}$ showing that the relative frequency of right versus left ventricular echogenic intracardiac focus was slightly over $90 \%$ on the left, $7 \%$ on the right, and the rest bilateral.

Earlier reports ${ }^{1-4}$ suggested that the echogenic intracardiac focus is a benign sonographic finding. Although Schechter et $\mathrm{al}^{2}$ concluded that it is totally benign, their study demonstrated that one of 26 fetuses (3.8\%) with this finding had trisomy 21 . Although this finding was not recognized as a sonographic feature of Down syndrome in 1987, subsequent reports ${ }^{5,6}$ have shown that an echogenic intracardiac focus may be associated with autosomal trisomies. Lehman et $\mathrm{al}^{5}$ found that $30 \%$ of fetuses with trisomy 13 had an echogenic intracardiac focus. Brown et al ${ }^{6}$ also described a fetus with trisomy 21 who had a calcified papillary muscle seen not only sonographically but also pathologically. Recently, Sepulveda et $\mathrm{al}^{7}$ showed a frequent association between an echogenic intracardiac focus and autosomal trisomies.

A large series ${ }^{8}$ in the pathology literature demonstrated that microcalcifications in the papillary muscle were characteristic features of fetuses with trisomies 13 and $21 ; 14$ of $85(16 \%)$ fetuses with trisomy 21 and seven of $18(39 \%)$ with trisomy 13 had a discrete calcification in the central papillary muscle. Six of $255(2 \%)$ control fetuses also had this finding. Our results confirm the pathologically based findings that $18 \%$ of fetuses with Down syndrome had an echogenic intracardiac focus seen sonographically, compared with $4.7 \%$ of fetuses without trisomy 21.

Our prevalence of trisomy 21 in fetuses with an echogenic intracardiac focus was distinctly different from other, smaller series. Petrikovsky et al $^{3}$ reported 41 fetuses with echogenic intracardiac focus, but none were aneuploid. In our high-risk population, $6.1 \%$ of fetuses with an echogenic intracardiac focus were noted to have Down syndrome, compared with $1.4 \%$ without an echogenic intracardiac focus. We did not include fetuses with trisomy 13 in our series because there was only one fetus diagnosed with trisomy 13 during the study period.

The use of a high-risk patient population with an elevated baseline incidence of karyotypic abnormalities is a recognized drawback to our study. However, this population was chosen because we could not obtain full karyotypic follow-up on this finite group of patients. Although we are unable to comment on the true meaning of an echogenic intracardiac focus in a low-risk population, we can extrapolate from our data as follows: The positive predictive value of an echogenic intracardiac focus for the detection of Down syndrome in a fetus of a woman 35 years of age (age-based risk of one in 250 ) is $1.5 \%$. For those considered at a lower age-based risk of one in 500 and one in 1000, the positive predictive value of this sonographic finding for identifying the presence of Down syndrome was 0.77 and $0.39 \%$, respectively. Our results show a significant increase risk of Down syndrome among fetuses with an echogenic intracardiac focus compared with those without this focus. The sensitivity of this sonographic finding is low, and most fetuses with an echogenic intracardiac focus are normal-both from a karyotypic and cardiac vicwpoint. Although the risk of aneuploidy is increased for fetuses with this sonographic finding, the revised positive predictive value for women with an age-based risk of one in 1000 for Down syndrome barely reached the accepted threshold for offering amniocentesis (positive predictive value $0.39 \%$ ). The revised risk should also depend on additional information, such as baseline age-based risk of the patient, 
maternal triple screen, and other sonographic features of the fetus.

There are a number of anatomic features of secondtrimester fetuses that, when identified sonographically, raise the risk that the fetus could have Down syndrome. We previously devised a scoring index that includes a thickened nuchal fold, short femur and humerus, mild pyelectasis, hyperechogenic bowel, and major structural abnormalities. ${ }^{9}$ It is difficult to determine exactly where the echogenic intracardiac focus fits in this scheme, without further study of low-risk patients. Based on our findings, we can speculate that although the presence of an echogenic intracardiac focus is associated with an increased risk for Down syndrome, the sensitivity is low (18\% of fetuses with Down syndrome had this sonographic feature). In our sonographic scoring system for the detection of Down syndrome, we assigned a score of 2 to abnormalities such as a thickened nuchal fold and major structural anomalies. A score of 1 was assigned to features such as a short femur or humerus, mild pyelectasis, hyperechogenic bowel, and choroid plexus cysts because these findings frequently occur among normal fetuses. ${ }^{9}$ If the echogenic intracardiac focus is given a score of 1 in young women otherwise at low risk for aneuploidy, more than one finding can be combined ( $\mathrm{eg}$, an echogenic intracardiac focus and mild hydronephrosis) before amniocentesis is recommended. Similarly, the risk of Down syndrome in fetuses carried by older women seeking to reduce their age-based risk of an affected fetus without amniocentesis may remain elevated if an echogenic intracardiac focus is detected. The finding of an echogenic intracardiac focus seen sonographically in the second trimester may prompt genetic counseling, although further study in low-risk populations will be necessary to fully evaluate the significance of this finding among younger women.

\section{References}

1. How HY, Villafane J, Parihus RR, Spinnato JA II. Small hyperechoic foci of the fetal cardiac ventricle: $A$ benign sonographic finding? Ultrasound Obstet Gynecol 1994:4:205-7.

2. Schechter AG, Fakhry J, Shapiro LR, Gewitz MH. In utero thickening of the chordae tendinae: A cause of intracardiac echogenic foci. ) Ultrasound Med 1987;6:691-5.

3. Petrikovsky BM, Challenger M, Wyse LJ. Natural history of echogenic foci within ventricles of the fetal heart. Ultrasound Obstet Gynecol 1995;5:92-4.

4. Levy DW, Mintz MC. The left ventricular echogenic focus: a normal finding. AJR 1988;150:85-6.

5. Lehman CD, Nyberg DA, Winter TC III, Kapur RP, Resta RG, Luthy DA. Trisomy 13 syndrome: Prenatal US findings in a review of 33 cases. Radiology 1995;194:217-22.

6. Brown DL, Roberts DJ, Miller WA. Left ventricular echogenic focus in the fetal heart: Pathologic correlation. J Ultrasound Med 1994;13: 613-6.

7. Sepulveda W, Cullen S, Nicolaidis P, Hollingsworth I, Fisk NM. Echogenic foci in the fetal heart: $\mathrm{A}$ marker of chromosomal abnormality. Br J Obstet Gynaecol 1995;102:490-2.

8. Roberts DJ, Genest D. Cardiac histologic pathology characteristic of trisomies 13 and 21. Hum Path 1992;23:1130-40.

9. Benacerraf BR, Nadel A, Bromley B. Identification of second trimester fetuses with autosomal trisomies using a sonographic scoring index. Radiology 1994:193:135-40.

Address reprint requests to:

Bryann Bromley, $M D$

Diagnostic Ultrasound Associates

333 Longwood Avenue

Boston, MA 02115

Received June 14, 1995.

Received in revised form August 11, 1995.

Accepted August 29, 1995.

Copyright 1995 by The American College of Obstetricians and Gynecologists 